\title{
Real-Space Renormalization and Energy-Level Statistics at the Quantum Hall Transition
}

\author{
Rudolf A. Römer ${ }^{1}$ and Philipp Cain ${ }^{2}$ \\ 1 Department of Physics and Centre for Scientific Computing, University of \\ Warwick, Coventry, CV4 7AL, United Kingdom \\ 2 Institut für Physik, Technische Universität Chemnitz, 09107 Chemnitz, \\ Germany
}

\begin{abstract}
We review recent applications of the real-space renormalization group (RG) approach to the integer quantum Hall $(\mathrm{QH})$ transition. The RG approach, applied to the Chalker-Coddington network model, reproduces the critical distribution of the power transmission coefficients, i.e., two-terminal conductances, $P_{\mathrm{c}}(G)$, with very high accuracy. The RG flow of $P(G)$ at energies away from the transition yields a value of the critical exponent, $\nu_{G}=2.39 \pm 0.01$, that agrees with most accurate large-size lattice simulations. Analyzing the evolution of the distribution of phases of the transmission coefficients upon a step of the RG transformation, we obtain information about the energy-level statistics (ELS). From the fixed point of the RG transformation we extract a critical ELS. Away from the transition the ELS crosses over towards a Poisson distribution. Studying the scaling behavior of the ELS around the QH transition, we extract the critical exponent $\nu_{\mathrm{ELS}}=2.37 \pm 0.02$.
\end{abstract}

The integer quantum Hall (QH) transition is described well in terms of a delocalization-localization transition of the electronic wavefunctions. In contrast to a usual metal-insulator transition (MIT), the QH transition is characterized by a single extended state located exactly at the center $\epsilon=0$ of each Landau band [1]. When approaching $\epsilon=0$, the localization length $\xi$ of the electron wavefunction diverges according to a power law $\epsilon^{-\nu}$, where $\epsilon$ defines the distance to the MIT for a suitable control parameter, e.g., the electron energy. On the theoretical side, the value of $\nu$ has been extracted from various numerical simulations, e.g., $\nu=2.5 \pm 0.5$ [2], $2.4 \pm 0.2$ [3], $2.35 \pm 0.03$ [4], and $2.39 \pm 0.01$ [5]. In experiments $\nu \approx 2.3$ has been obtained, e.g., from the frequency [6] or the sample size [7] dependence of the critical behavior of the resistance in the transition region at strong magnetic field.

Recently, a semianalytical description of the integer QH transition, based on the extension of the scaling ideas for the classical percolation [8] to the Chalker-Coddington (CC) model of the quantum percolation [2], has been developed $[9,10]$. The key idea of this description, a real-space-renormalization group (RG) approach, is the following. Each RG step corresponds to a doubling of the system size. The RG transformation relates the conductance distribution of the sample at the next step to the conductance distribution at the previous step. The fixed point (FP) of this transformation, yields the distribution of the conductance, $P_{\mathrm{c}}(G)$, of a macroscopic sample at the $\mathrm{QH}$ 
transition. This universal distribution describes the mesoscopic properties of a fully coherent QH sample. Analogously to the classical percolation [8], the correlation length exponent, $\nu$, was extracted from the RG procedure [5] using the fact that a slight shift of the initial distribution with respect to the FP distribution $P_{\mathrm{c}}(G)$ drives the system to the insulator upon renormalization. Then the rate of the shift of the distribution maximum determines the value of $\nu$. Remarkably, both $P_{\mathrm{c}}(G)$ and the critical exponent obtained within the RG approach $[5,11,12]$ agree very well with the "exact" results of the large-scale simulations $[3,4,13-15]$.

The goal of the present paper is threefold. First, we briefly review the basic ingredients that constitute the real-space RG method in the QH situation [5]. Second, we extend the RG approach to include the level statistics at the QH transition and apply a method analogous to the finite-size-corrections analysis to extract $\nu$ from the energy-level statistics (ELS) obtained within the RG approach. This method yields $\nu=2.37 \pm 0.02$, which is even closer to the most precise large-scale simulations result $\nu=2.35 \pm 0.03$ [4] than the value $\nu=2.39 \pm 0.01$ inferred from the conductance distribution [5]. This agreement is by no means trivial. Indeed, the original RG transformation [5] related the conductances, i.e., the absolute values of the transmission coefficients of the original and the doubled samples, while the phases of the transmission coefficients were assumed random and uncorrelated. In contrast, the level statistics at the transition corresponds to the FP in the distribution of these phases. Therefore, the success of the RG approach for conductances does not guarantee that it will be equally accurate quantitatively for the level statistics. Third, we show that the RG structure employed in the present approach, which is constructed from 5 saddle points (SP), represents in many aspects the minimal model of the $\mathrm{QH}$ transition. A further reduction in the number of SP leads to less reliable results.

\section{The RG approach to the CC model}

Our RG approach to the QH transition $[5,9,10]$ is based on the RG unit shown in Fig. 1. The unit is a fragment of the CC network consisting of five nodes. Each node, $i$, is characterized by the transmission coefficient $t_{i}$, which is an amplitude to deflect an incoming electron along the link to the left. Analogously, the reflection coefficient $r_{i}=\left(1-t_{i}^{2}\right)^{1 / 2}$ is the amplitude to deflect the incoming electron to the right. Doubling of the sample size corresponds to the replacement of the RG unit by a single node. The RG transformation expresses the transmission coefficient of this effective node, $t^{\prime}$, through the transmission coefficients of the five constituting nodes [9]

$$
t^{\prime}=\left|\frac{t_{1} t_{5}\left(r_{2} r_{3} r_{4} e^{i \Phi_{2}}-1\right)+t_{2} t_{4} e^{i\left(\Phi_{3}+\Phi_{4}\right)}\left(r_{1} r_{3} r_{5} e^{-i \Phi_{1}}-1\right)}{\left(r_{3}-r_{2} r_{4} e^{i \Phi_{2}}\right)\left(r_{3}-r_{1} r_{5} e^{i \Phi_{1}}\right)+\left(t_{3}-t_{4} t_{5} e^{i \Phi_{4}}\right)\left(t_{3}-t_{1} t_{2} e^{i \Phi_{3}}\right)}\right|
$$




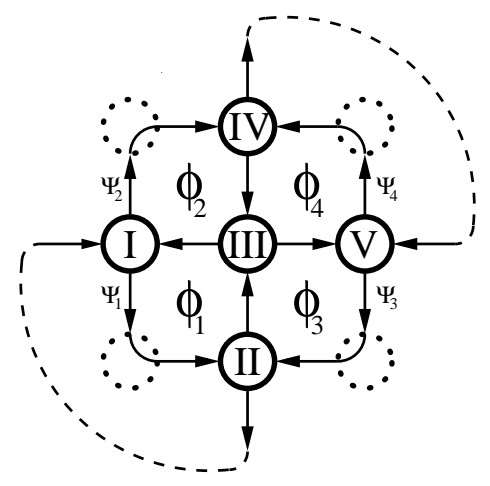

Fig. 1. Chalker-Coddington network on a square lattice consisting of nodes (circles) and links (arrows). The RG unit used to construct the matrix (4) combines five nodes (full circles) by neglecting some connectivity (dashed circles). $\Phi_{1}, \ldots, \Phi_{4}$ are the phases acquired by an electron along the loops as indicated by the arrows. $\Psi_{1}, \ldots, \Psi_{4}$ represent wave function amplitudes, and the thin dashed lines illustrate the boundary conditions used for the computation of level statistics

Here $\Phi_{j}$ are the phases accumulated along the closed loops (Fig. 1). Within the RG approach to the conductance distribution, information about electron energy is incorporated only into the values of $t_{i}$ [5]. The energy dependence of phases, $\Phi_{j}$, is irrelevant; they are assumed completely random. Due to this randomness, the transmission coefficients, $t_{i}$, for a given energy, are also randomly distributed with a distribution function $P(t)$. Then the transformation (1) allows, upon averaging over $\Phi_{j}$, to generate the next-step distribution $P\left(t^{\prime}\right)$. Therefore, within the RG scheme, a delocalized state corresponds to the FP distribution $P_{\mathrm{c}}(t)$ of the RG transformation. Due to the symmetry of the RG unit, it is obvious that the critical distribution, $P_{\mathrm{c}}\left(t^{2}\right)$, of the power transmission coefficient, $t^{2}=G$, which has the meaning of the two-terminal conductance, is symmetric with respect to $t^{2}=\frac{1}{2}$ as shown in Fig. 2. In other words, the RG transformation respects the duality between transmission and reflection. The critical distribution $P_{\mathrm{c}}(G)$ found in Refs. [5] and [9] agrees very well with the results of direct large-scale simulations.

\section{RG Approach to the ELS}

It has been realized long ago that, alongside with the change in the behavior of the eigenfunctions, a localization-delocalization transition manifests itself in the statistics of the energy levels. In particular, as the energy is swept across the mobility edge, the shape of the ELS crosses over from the WignerDyson distribution, corresponding to the appropriate universality class, to the Poisson distribution. Moreover, finite-size corrections to the critical ELS close to the mobility edge allow to determine the value of the correlation length exponent [16], thus avoiding an actual analysis of the spatial extent 


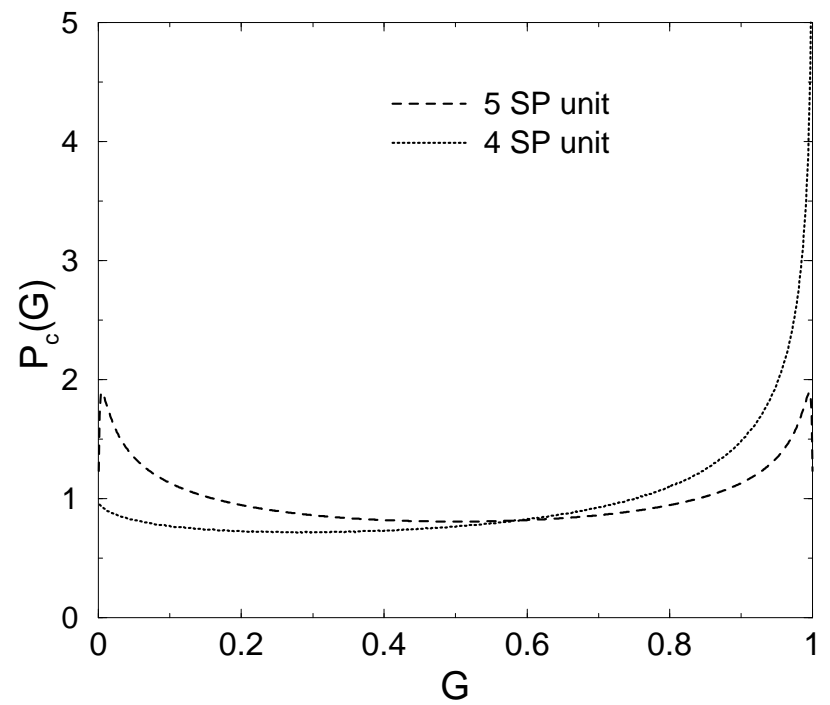

Fig. 2. The critical distribution of the conductance $P_{\mathrm{c}}(G)$ at the $\mathrm{QH}$ transition obtained using the $5 \mathrm{SP}$ RG unit (dashed line). The dotted line denotes a $4 \mathrm{SP}$ RG unit as discussed in Sect. 4. The latter distribution clearly deviates from the expected symmetry with respect to $G=0.5$

of the wave functions. For this reason, the ELS constitutes an alternative to the MacKinnon-Kramer [17-20] and to the transmission-matrix [21,22] approaches to the numerical study of localization.

\subsection{Derivation of the Network Operator for the RG}

As has been shown by Fertig [23], energy levels of an 2D CC network can be computed from the energy dependence of the so called network operator $U(E) . U$ is constructed similar to the system of equations for obtaining the transmission coefficient $t^{\prime}$ of the RG unit as presented in Eq. (1). Every SP of the network contributes two scattering equations. Each of them describes the amplitude of one outgoing channel using the amplitudes of the two incoming channels weighted by the transmission and reflection coefficients $t$ and $r$ in which also the random phase $\Phi$ of the links between SP's can be incorporated. When comparing to the calculation of the transmission coefficient $t^{\prime}$ an essential difference has to be taken into account. Energy levels are defined only in a closed system which requires to apply appropriate boundary conditions. The energy dependence of $U(E)$ enters trough the energy dependence of the $t_{i}(E)$ of the SP's, as well as the energy dependence of the phases $\Phi_{j}(E)$ of 
the links. Considering the vector $\Psi$ of wave amplitudes on the links of the network, the eigenenergies can now be obtained from the stationary condition

$$
U(E) \Psi=\Psi .
$$

Nontrivial solutions exist only for discrete energies $E_{k}$, which coincide with the eigenenergies of the system [23]. The evaluation of the $E_{k}$ 's according to Eq. (2) is numerically very expensive. For that reason a simplification was proposed [24]. Instead of solving the real eigenvalue problem, calculating a spectrum of quasienergies $\omega$ is suggested following from

$$
U(E) \Psi_{l}=e^{i \omega_{l}(E)} \Psi_{l} .
$$

For fixed energy $E$ the $\omega_{l}$ are expected to obey the same statistics as the real eigenenergies [24]. This approach makes is perfectly suited for large-size numerical simulations, e.g. studying $50 \times 50$ SP networks.

In order to combine the above algorithm with the RG iteration, in which a rather small unit of SP's is considered, we first "close" the RG unit at each RG step in order to discretize the energy levels as shown in Fig. 1 with dashed lines. For a given closed RG unit with a fixed set of $t_{i}$-values at the nodes, the positions of the energy levels are determined by the energy dependences, $\Phi_{j}(E)$, of the four phases along the loops. These phases change by $\sim \pi$ within a very narrow energy interval, inversely proportional to the sample size. Within this interval the change of the transmission coefficients is negligibly small. A closed RG unit in Fig. 1 contains 10 links, and, thus, it is described by 10 amplitudes. Each link is characterized by an individual phase. On the other hand, it is obvious that the energy levels are determined only by the phases along the loops. One way to derive $U$ is to combine the individual phases into phases $\Phi_{j}$ connected to the four inner loops of the unit and to exclude from the original system of 10 equations all amplitudes except the "boundary" amplitudes $\Psi_{j}$ (Fig. 1). The network operator for the remaining four amplitudes is a $4 \times 4$ matrix $\left\{U_{n m}\right\}$ with elements

$$
\begin{array}{ll}
U_{11}=\left(r_{1} r_{2}-t_{1} t_{2} t_{3}\right) e^{-i \Phi_{1}} & U_{12}=\left(t_{1} r_{2}+t_{2} t_{3} r_{1}\right) e^{-i \Phi_{1}} \\
U_{13}=t_{2} t_{5} r_{3} e^{-i \Phi_{1}} & U_{14}=t_{2} r_{3} r_{5} e^{-i \Phi_{1}} \\
U_{21}=-t_{1} r_{3} r_{4} e^{-i \Phi_{2}} & U_{22}=r_{1} r_{3} r_{4} e^{-i \Phi_{2}} \\
U_{23}=-\left(t_{4} r_{5}+t_{3} t_{5} r_{4}\right) e^{-i \Phi_{2}} & U_{24}=\left(t_{4} t_{5}-t_{3} r_{4} r_{5}\right) e^{-i \Phi_{2}} \\
U_{31}=-t_{1} t_{4} r_{3} e^{-i \Phi_{4}} & U_{32}=t_{4} r_{1} r_{3} e^{-i \Phi_{4}} \\
U_{33}=\left(r_{4} r_{5}-t_{3} t_{4} t_{5}\right) e^{-i \Phi_{4}} & U_{34}=-\left(t_{5} r_{4}+t_{3} t_{4} r_{5}\right) e^{-i \Phi_{4}} \\
U_{41}=-\left(t_{2} r_{1}+t_{1} t_{3} r_{2}\right) e^{-i \Phi_{3}} & U_{42}=-\left(t_{1} t_{2}-t_{3} r_{1} r_{2}\right) e^{-i \Phi_{3}} \\
U_{43}=t_{5} r_{2} r_{3} e^{-i \Phi_{3}} & U_{44}=r_{2} r_{3} r_{5} e^{-i \Phi_{3}}
\end{array}
$$

which can be substituted in Eq. (3). Then the energy levels, $E_{k}$, of the closed RG unit including phases $\Phi_{j}(E)=\Phi_{j}\left(E_{k}\right)$, are the energies for which one of the four eigenvalues of the matrix $U$ is equal to one. Thus, the calculation of the energy levels reduces to a diagonalization of the $4 \times 4$ matrix. 
The crucial step now is the choice of the energy dependence $\Phi_{j}(E)$. If each loop in Fig. 1 is viewed as a closed equipotential as it is the case for the first step of the RG procedure [2], then $\Phi_{j}(E)$ is a true magnetic phase and changes linearly with energy with a slope governed by the actual potential profile, which, in turn, determines the drift velocity. Thus

$$
\Phi_{j}(E)=\Phi_{0, j}+2 \pi \frac{E}{s_{j}},
$$

where a random part, $\Phi_{0, j}$, is uniformly distributed within $[0,2 \pi]$, and $2 \pi / s_{j}$ is a random slope. Here the coefficient $s_{j}$ acts as an initial level spacing connected to the loop $j$ of the RG unit by defining a periodicity of the corresponding phase. Strictly speaking, the dependence (5) applies only for the first RG step. At each following step, $n>1, \Phi_{j}(E)$ is a complicated function of $E$ which carries information about all energy scales at previous steps. However, in the spirit of the RG approach, one can assume that $\Phi_{j}(E)$ can still be linearized within a relevant energy interval. The conventional RG approach suggests that different scales in real space can be decoupled. Linearization of Eq. (5) implies a similar decoupling in energy space.

With $\Phi_{j}(E)$ given by Eq. (5), the statistics of energy levels determined by the matrix equation (3) is obtained by averaging over the random initial phases $\Phi_{0, j}$ and values $t_{i}$ chosen randomly according to a distribution $P(t)$. For every realization the levels $E_{k}$ are computed from the solutions $\omega\left(E_{k}\right)=0$ of Eq. (3) yielding 3 level spacings as illustrated in Fig. 3. Thus the situation is comparable with estimating the true random matrix ensemble distribution functions from small, say, $2 \times 2$ matrices only $[25,26]$. Within the RG approach, the slopes $s_{j}$ as in Eq. (5) determine the level spacings at the first step. They are randomly distributed with a distribution function $P_{0}(s)$. Subsequent averaging over many realizations yields the ELS, $P_{1}(s)$, at the second step. Then the key element of the RG procedure, as applied to the level statistics, is using $P_{1}(s)$ as a distribution of slopes in Eq. (5). This leads to the next-step ELS and so on.

The approach of this work relies on the real eigenenergies of the RG unit. The simpler computation of the spectrum of quasienergies adopted in largescale simulations within the CC model $[24,27]$ cannot be applied since the energy dependence of phases $\Phi_{j}$ in the elements of the matrix is neglected and only the random contributions, $\Phi_{0, j}$, are kept. Nevertheless it is instructive to compare the two procedures as presented in Fig. 3. The figure shows the dependence of the 4 quasienergies $\omega_{k}$ on the energy $E$ calculated for two single sample RG units, with $t_{i}$ chosen from the critical distribution $P_{\mathrm{c}}(t)$. The energy dependence of the phases $\Phi_{j}$ was chosen from the ELS of the unitary random matrix ensemble (GUE) according to Eq. (5). It is seen that the dependences $\omega(E)$ range from remarkably linear and almost parallel (Fig. 3a) to strongly nonlinear (Fig. 3b). 

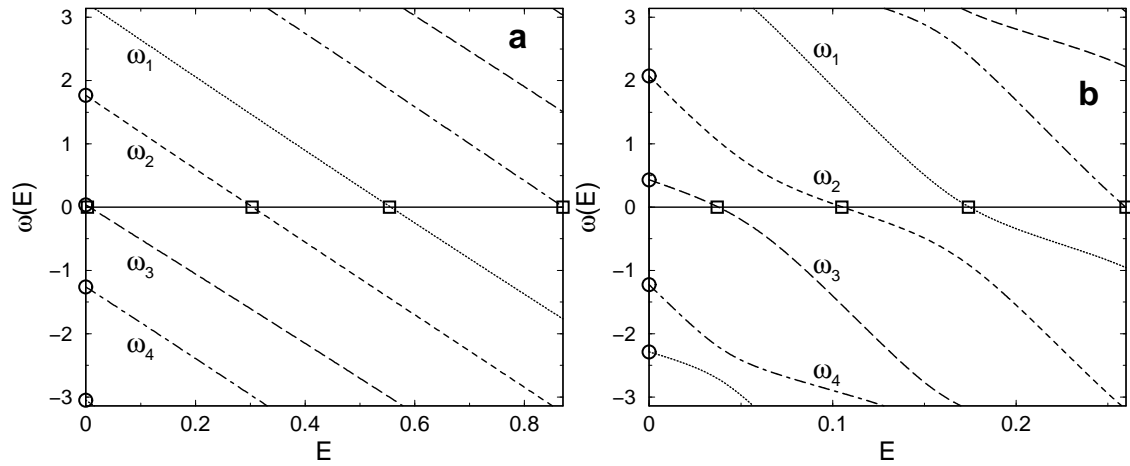

Fig. 3. Energy dependence of the quasieigenenergies $\omega$ for two sample configurations. Instead of using the quasispectrum obtained from $\omega_{l}(E=0)(\bigcirc)$ we calculate the real eigenenergies according to $\omega\left(E_{k}\right)=0(\square)$. Different line styles distinguish different $\omega_{l}(E)$. We emphasize that the observed behavior varies from sample to sample between remarkably linear (a) and strongly nonlinear (b)

\subsection{The Shape of the ELS at the QH Transition}

First, let us turn our attention to the shape of the ELS at the QH transition. As starting distribution $P_{0}(s)$ of the RG iteration, we choose the ELS of GUE, since previous simulations [24,28] indicate that the critical ELS is close to GUE. According to $P_{0}(s), s_{j}$ is drawn randomly and $\Phi_{j}, j=1, \ldots, 4$ is set as in Eq. (5). For the transmission coefficients of the SP the FP distribution $P_{\mathrm{c}}(t)$, obtained in Sect. 1, is used as initial distribution $P_{0}(t)$. And from $P_{0}(t)$, the $5 t_{i}, i=1, \ldots, 5$, are selected. As in Sect. 1 the RG transformation (1) is used to compute $10^{7}$ super-transmission coefficients $t^{\prime}$. The accumulated distribution $P_{1}\left(t^{\prime}\right)$ is again discretized in at least 1000 bins, such that the bin width is typically 0.001 for the interval $t \in[0,1] . P_{1}\left(t^{\prime}\right)$ is then smoothed by a Savitzky-Golay filter [29] in order to decrease statistical fluctuations. By finding solutions $\omega\left(E_{k}\right)=0$ of Eq. (3) the new ELS $P_{1}\left(s^{\prime}\right)$ is constructed from the "unfolded" energy-level spacings $s_{m}^{\prime}=\left(E_{m+1}-E_{m}\right) / \Delta$, where $m=1,2,3, E_{k+1}>E_{k}$ and the mean spacing $\Delta=\left(E_{4}-E_{1}\right) / 3$. Due to the "unfolding" [30] with $\Delta$, the average spacing is set to one for each sample and in each RG-iteration step spacing data of $2 \times 10^{6}$ super-SP's can be superimposed. The resulting ELS is discretized in bins with largest width 0.01 . In the following iteration step the procedure is repeated using the $P_{1}$ 's as initial distributions. Convergence of the iteration process is assumed when the mean-square deviations of both distributions $P_{n}(t)$ and $P_{n}(s)$ deviate by less than $10^{-4}$ from predecessors $P_{n-1}(t)$ and $P_{n-1}(s)$. Once the (unstable) FP has been reached, the $P_{n}$ 's should in principle remain unchanged during all further RG iterations. Our simulations show [5] that unavoidable numerical inaccuracies sum up within several further iterations and lead to a drift away from the FP. In order to stabilize our calculation, we therefore use in every 
RG step instead of $P_{n}(t)$ the FP distribution $P_{\mathrm{c}}(t)$. This trick does not alter the results but speeds up the convergence of the RG for $P_{\mathrm{c}}(s)$ considerably.

This now enables us to determine the critical ELS $P_{\mathrm{c}}(s)$. The RG iteration converges rather quickly after only $2-3 \mathrm{RG}$ steps. The resulting $P_{\mathrm{c}}(s)$ is shown in Fig. 4 together with the ELS for GUE. $P_{\mathrm{c}}(s)$ exhibits the expected

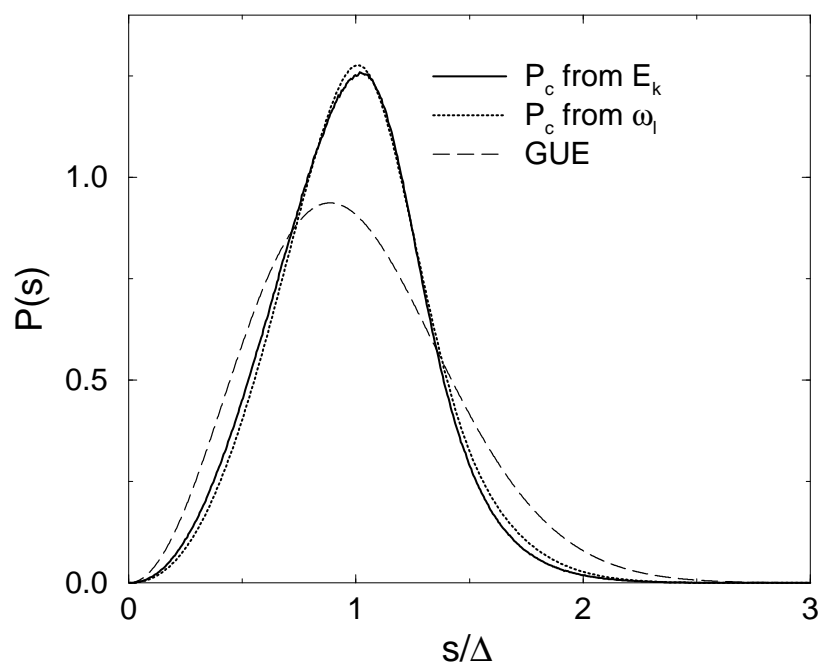

Fig. 4. FP distributions $P_{\mathrm{c}}(s)$ obtained from the spectrum of $\omega_{l}(E=0)$ and from the RG approach using the real eigenenergies $E_{k}$ in comparison to the ELS for GUE. As in all other graphs $P(s)$ is shown in units of the mean level spacing $\Delta$

features, namely, level repulsion for small $s$ and a long tail at large $s$, but the overall shape of $P_{\mathrm{c}}(s)$ differs noticeably from GUE. In the previous large-size lattice simulations $[24,28]$ the obtained critical ELS was much closer to GUE than $P_{\mathrm{c}}(s)$ in Fig. 4. This fact, however, does not reflect on the accuracy of the RG approach. Indeed, as it was demonstrated recently, the critical ELS - although being system size independent - nevertheless depends on the geometry of the samples [31] and on the specific choice of boundary conditions $[32,33]$. Sensitivity to the boundary conditions does not affect the asymptotics of the critical distribution, but rather manifests itself in the shape of the "body" of the ELS. Recall now that the boundary conditions which have been imposed to calculate the energy levels (dashed lines in Fig. 1) are non-periodic.

As mentioned above, the critical ELS has also been computed previously by diagonalizing $U(0)$ and studying the distribution of quasienergies. In Fig. 4 the result of this procedure using the present RG approach is shown. It appears that the resulting distribution is almost identical to $P_{\mathrm{c}}(s)$. This ob- 

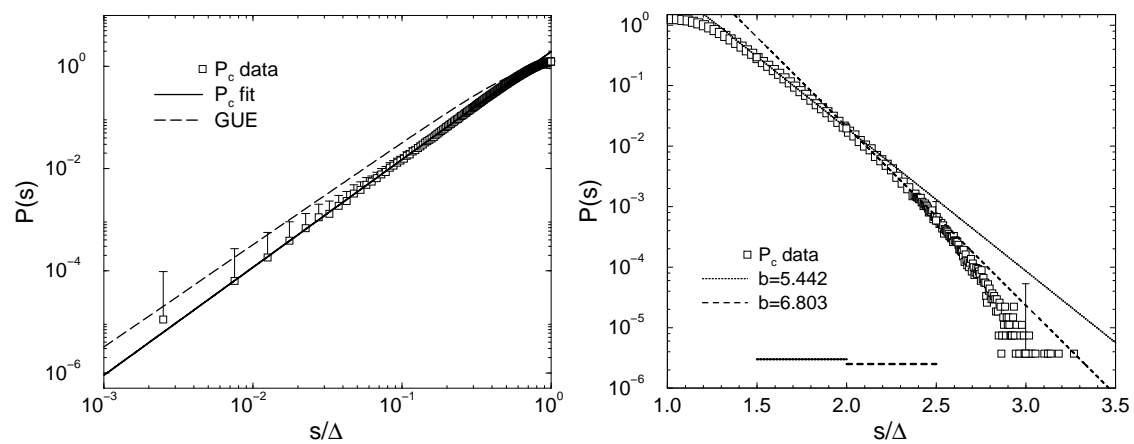

Fig. 5. Left: FP $P_{\mathrm{c}}(s)$ for small $s$ in agreement with the predicted $s^{2}$ behavior. Due to the log-log plot errors are shown in the upper direction only. Right: The large $s$ tail of $P_{\mathrm{c}}(s)$ compared with fits according to the predictions of Ref. [16] (lines). The interval used for fitting is indicated by the bars close to the lower axis. For clarity errors are shown in upper direction and for $s / \Delta=1.5,2.0,2.5,3.0$ only. For $s / \Delta<2.4$, only every 5 th data point is drawn by a symbol

servation is highly non-trivial, since, as follows from Fig. 3, there is no simple relation between the energies and quasienergies.

\subsection{Small and Large $s$ Behavior}

As we have seen, the general shape of the critical ELS is not universal. However, the small-s behavior of $P_{\mathrm{c}}(s)$ must be the same as for GUE, namely $P_{\mathrm{c}}(s) \propto s^{2}$. This is because delocalization at the $\mathrm{QH}$ transition implies level repulsion $[16,24,27,28,34-42]$. In Fig. 5 we show that this is also true for the RG approach. The given error bars of our numerical data are standard deviations computed from a statistical average of $100 \mathrm{FP}$ distributions each obtained for different random sets of $t_{i}$ 's and $\Phi_{j}$ 's within the RG unit. In general, within the RG approach, the $s^{2}$-asymptotics of $P(s)$ is most natural. This is because the levels are found from diagonalization of the $4 \times 4$ unitary matrix with absolute values of elements widely distributed between 0 and 1 . The right form of the large- $s$ tail of $P(s)$ is Poissonian, $P_{\mathrm{c}}(s) \propto \exp (-b s)$ [16] for $s \geq 3 \Delta$. The data has a high accuracy only for $s / \Delta \leq 2.5$. For such $s$, the distribution $P_{\mathrm{c}}(s)$ does not yet reach its large- $s$ tail and the fit parameters shown in Fig. 5 depend largely on the $s$-interval chosen.

\section{$3 \quad$ Scaling Results for the ELS}

\subsection{Finite-Size Scaling at the QH Transition}

The critical exponent $\nu$ of the $\mathrm{QH}$ transition governs the divergence of the correlation length $\xi_{\infty}$ as a function of the control parameter $z_{0}$, i.e.

$$
\xi_{\infty}\left(z_{0}\right) \propto\left|z_{0}-z_{\mathrm{c}}\right|^{-\nu},
$$


where $z_{\mathrm{c}}$ is the critical value. For the $\mathrm{QH}$ transition $\nu \approx 2.35$ has been calculated by a variety of numerical methods [2-4] and is in agreement with the experimental estimates $\nu \approx 2.3[6,43,44]$. As presented in Sect. 1 the $\mathrm{RG}$ approach for the conductance distribution yields a rather accurate value $\nu=2.39 \pm 0.01$. In order to extract $\nu$ from the ELS the one-parameterscaling hypothesis [45] is employed. This approach describes the rescaling of a quantity $\alpha\left(N ;\left\{z_{i}\right\}\right)$ - depending on (external) system parameters $\left\{z_{i}\right\}$ and the system size $N$ - onto a single curve by using a scaling function $f$

$$
\alpha\left(N ;\left\{z_{i}\right\}\right)=f\left(\frac{N}{\xi_{\infty}\left(\left\{z_{i}\right\}\right)}\right) .
$$

Since Eq. (6), as indicated by " $\infty$ ", holds only in the limit of infinite system size, we now use the scaling assumption to extrapolate $f$ to $N \rightarrow \infty$ from the finite-size results of the computations. The knowledge about $f$ and $\xi_{\infty}$ then allows to derive the value of $\nu$.

We use the natural parametrization $t=\left(e^{z}+1\right)^{-1 / 2}[9]$, such that $z$ can be identified with a dimensionless electron energy. The universal conductance distribution at the transition, $P_{\mathrm{c}}(G)$, corresponds to a distribution $Q_{\mathrm{c}}(z)[5]$ which is symmetric with respect to $z=0$ and has a shape close to a Gaussian. The RG procedure for the conductance distribution converges and yields $Q_{\mathrm{c}}(z)$ only if the initial distribution is an even function of $z$. This suggests to choose as a control parameter in Eq. (7), the position $z_{0}$ of the maximum of the function $Q(z)$. The meaning of $z_{0}$ is an electron energy measured from the center of the Landau band. The fact that the $\mathrm{QH}$ transition is infinitely sharp implies that for any $z_{0} \neq 0$, the $\mathrm{RG}$ procedure drives the initial distribution $Q\left(z-z_{0}\right)$ towards an insulator, either with complete transmission of the network nodes (for $z_{0}>0$ ) or with complete reflection of the nodes (for $\left.z_{0}<0\right)$.

\subsection{Scaling for $\alpha_{\mathrm{P}}$ and $\alpha_{\mathrm{I}}$}

In principle, one is now free to choose for the finite-size scaling analysis (FSS) any characteristic quantity $\alpha\left(N ; z_{0}\right)$ constructed from the ELS which has a systematic dependence on system size $N$ for $z_{0} \neq 0$ while being constant at the transition $z_{0}=0$. Because of the large number of possible choices $[16,28,46-49]$ a restriction to two appropriate quantities is made which are obtained by integration of the ELS and have already been successfully used in Refs. [46,50], namely

$$
\alpha_{\mathrm{P}}=\int_{0}^{s_{0}} P(s) d s, \quad \text { and } \quad \alpha_{\mathrm{I}}=\frac{1}{s_{0}} \int_{0}^{s_{0}} I(s) d s,
$$

with $I(s)=\int_{0}^{s} P\left(s^{\prime}\right) d s^{\prime}$. The integration limit is chosen as $s_{0}=1.4$ which approximates the common crossing point [46] of all ELS curves as can be seen in Fig. 6. Thus $P\left(s_{0}\right)$ is independent of the distance $\left|z-z_{\mathrm{c}}\right|$ to the critical 


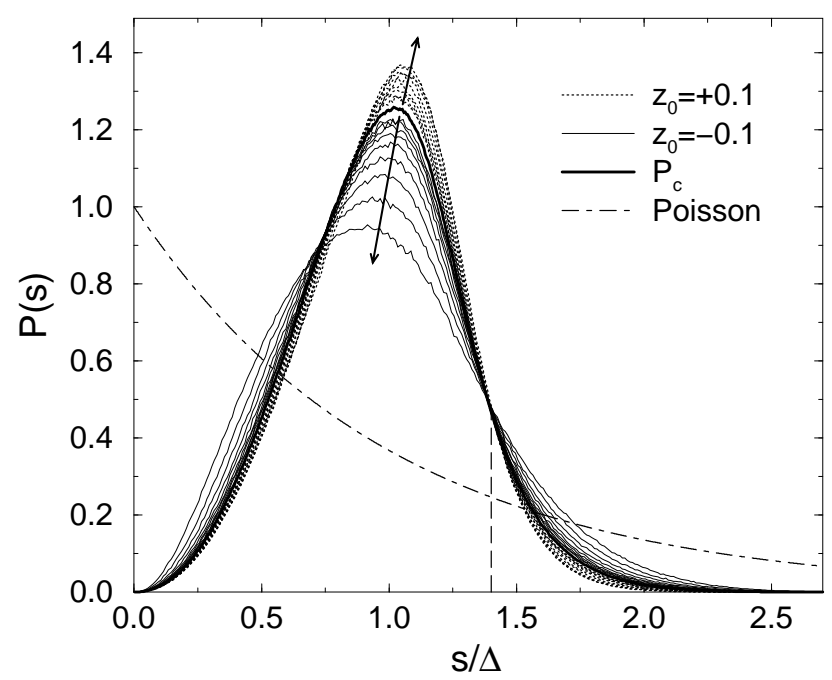

Fig. 6. RG of the ELS used for the computation of $\nu$. The dotted lines corresponds to the first 9 RG iterations with an initial distribution $P_{0}$ shifted to the metallic regime $\left(z_{0}=0.1\right)$ while the thin full lines represent results for a shift toward localization $\left(z_{0}=-0.1\right)$. Within the RG procedure the ELS moves away from the $\mathrm{FP}$ as indicated by the arrows. At $s / \Delta \approx 1.4$ the curves cross at the same point a feature we exploit when deriving a scaling quantity from the ELS

point and the system size magnification $N$. Since $\alpha_{\mathrm{I}, \mathrm{P}}\left(N, z_{0}\right)$ is analytical for finite $N$, one can expand the scaling function $f$ at the critical point. The first order approximation yields

$$
\alpha\left(N, z_{0}\right) \sim \alpha\left(N, z_{\mathrm{c}}\right)+a\left|z_{0}-z_{\mathrm{c}}\right| N^{1 / \nu}
$$

where $a$ is a coefficient. For our calculation we use higher order expansions [51] expanding $f$ twice, first, in terms of Chebyshev polynomials of order $\mathcal{O}_{\nu}$ and, second, as Taylor expansion with terms $\left|z_{0}-z_{c}\right|$ in the order $\mathcal{O}_{z}$. This procedure allows to describe deviations from linearity in $\left|z_{0}-z_{c}\right|$ at the transition. Contributions from an irrelevant scaling variable can be neglected since the transition point $z_{0}=0$ is known. In Fig. 7 the resulting fits for $\alpha_{\mathrm{P}}$ at the transition are shown.

The fits are chosen in a way such that the total number of parameters is kept at a minimal value and the fit agrees well with the numerical data. The corresponding scaling curves for $\alpha_{\mathrm{P}}$ are displayed in Fig. 7. In the plots the two branches for complete reflection $\left(z_{0}<0\right)$ and complete transmission $\left(z_{0}>0\right)$ can be distinguished clearly. In order to estimate the error of the fitting procedure the results for $\nu$ obtained by different orders $\mathcal{O}_{\nu}$ and $\mathcal{O}_{z}$ of the expansion, system sizes $N$, and regions around the transition are compared. A part of the over 100 fit results together with the standard deviation of the fit are given in Ref. [52]. The value of $\nu$ is calculated as average of all 

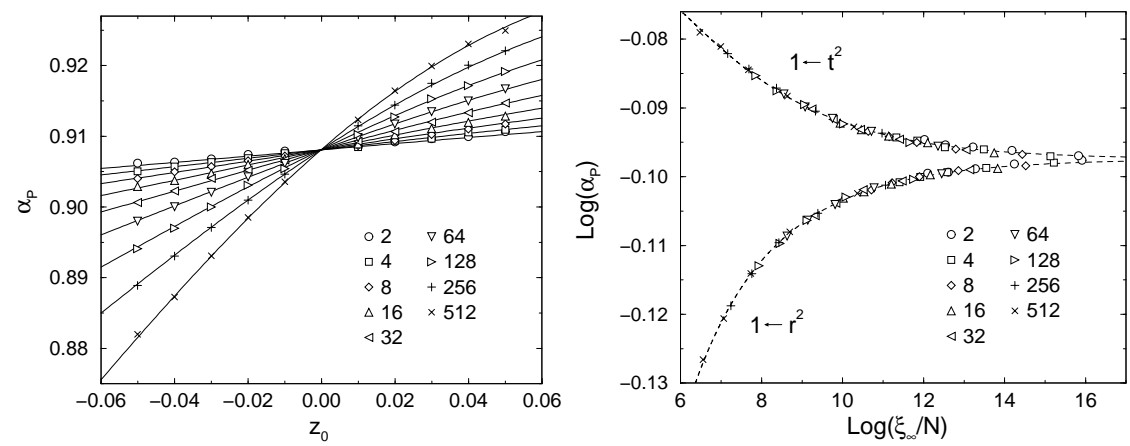

Fig. 7. Left: Behavior of $\alpha_{\mathrm{P}}$ at the $\mathrm{QH}$ transition as results of the RG of the ELS. Data are shown for RG iterations $n=1, \ldots, 9$ corresponding to effective system sizes $N=2^{n}=2, \ldots, 512$. Full lines indicate the functional dependence according to FSS using the $\chi^{2}$ minimization with $\mathcal{O}_{\nu}=2$ and $\mathcal{O}_{z}=3$. Right: FSS curves resulting from the $\chi^{2}$ fit of our data shown in Fig. 7. Different symbols correspond to different effective system sizes $N=2^{n}$. The data points collapse onto a single curve indicating the validity of the scaling approach

individual fits where the resulting error of $\nu$ was smaller than 0.02 resulting in $\nu=2.37 \pm 0.02$. This is in excellent agreement with the previously quoted results $[2-5]$.

\section{Test of Different SP Unit}

Apparently, the quality of the RG approach crucially depends on the choice the RG unit. For the construction of a proper chosen RG unit two conflicting aspects have to be considered. (i) With the size of the RG unit also the accuracy of the RG approach increases since the RG unit can preserve more of the connectivity of the original network. (ii) As a consequence of larger RG units the computational effort for solving the scattering problem rises, especially in the case where an analytic solution, as Eq. (1), is not attained. Because of these reasons building an RG unit is an optimization problem depending mainly on the computational resources available. As mentioned in the previous Section larger RG units were already studied in $[11,12,53]$. In these works the authors could not benefit from an analytic solution and achieve only a similar and less accurate statistics in comparison with the results presented here. In this Section the opposite case is studied using a small RG unit proposed in Ref. [54] in the context of the Hall resistivity.

The super-SP now consists only of 4 SP's as shown in Fig. 8. It resembles the 5 SP's unit (Fig. 1) used previously leaving out the SP in the middle of 


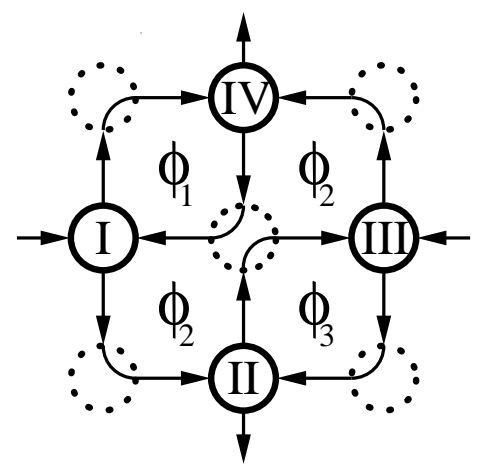

Fig. 8. RG unit constructed from 4 SP's indicated by full circles. Some connectivity is neglected (dotted circles). The phases $\Phi_{j}$ are accumulated by the electron motion (arrows) along contours of the energy potential

the structure. Again the scattering problem can be formulated as a system of now 8 equations which is solved analytically

$$
t_{4 \mathrm{SP}}^{\prime}=\left|\frac{t_{2} t_{3} e^{i \Phi_{2}}\left(r_{1} r_{4} e^{-i \Phi_{1}}-1\right)+t_{1} t_{4}\left(r_{2} r_{3} e^{i \Phi_{3}}-1\right)}{\left(1-r_{2} r_{3} e^{i \Phi_{3}}\right)\left(1-r_{1} r_{4} e^{i \Phi_{1}}\right)+t_{1} t_{2} t_{3} t_{4} e^{i \Phi_{2}}}\right| .
$$

The result can be verified using Eq. (1) after setting $t_{3}=0$ and $r_{3}=1$, joining the phases $\Phi_{1}$ and $\Phi_{4}$ and renumbering the indices.

The RG transformation (10) is then applied within the RG approach analogously to the $5 \mathrm{SP}$ unit. First the FP distribution $P_{\mathrm{c}}(G)$ is obtained. A comparison of $P_{\mathrm{c}}(G)$ for both RG units is shown in Fig. 2. In the case of the 5 SP unit the FP distribution $P_{\mathrm{c}}(G)$ exhibits a flat minimum around $G=0.5$, and sharp peaks close to $G=0$ and $G=1$. It is symmetric with respect to $G \approx 0.5$. The $4 \mathrm{SP}$ unit yields differing results. While $P_{\mathrm{c}}(G)$ is still rather flat it is clearly asymmetric, which already indicates that the $4 \mathrm{SP}$ unit can not describe all of the underlying symmetry of the CC network.

The $P_{\mathrm{c}}(G)$ for the $4 \mathrm{SP}$ unit is then used in the calculation of the critical exponent $\nu$ to construct the shifted initial distributions $Q_{0}(z)$. The behavior of $\nu$ as function of $n$ for the 4 and 5 SP RG units is demonstrates in Fig. 9. Both curves approach convergence monotonously from larger values of $\nu$. During all iteration steps, $\nu$ for the $4 \mathrm{SP}$ differs from the $5 \mathrm{SP}$ result by an almost constant positive shift. After 8 iterations, which equals an increase of system size by a factor of 256, one finds $\nu_{5 \mathrm{SP}}=2.39 \pm 0.01$ and $\nu_{4 \mathrm{SP}}=$ $2.74 \pm 0.02$. The error describes a confidence interval of $95 \%$ as obtained from the fit to a linear behavior. The result for $\nu_{4 \mathrm{SP}}$ deviates clearly from the five SP result and also from the values obtained by other methods [2-4]. In addition to these findings also the discussion in Sect. 1 indicates that the 4 SP RG unit fails to describe the critical properties at the $\mathrm{QH}$ transition 


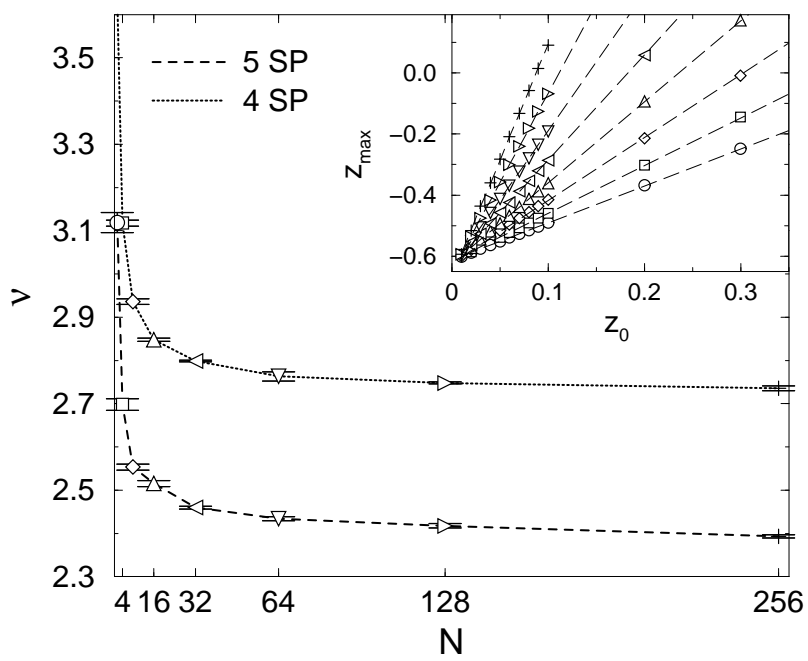

Fig. 9. The critical exponent $\nu$ as function of the effective system size $N=2^{n}$ for $4 \mathrm{SP}$ (dotted line) and $5 \mathrm{SP}$ unit (dashed line). Inset: Maximum $z_{\max }$ of $Q(z)$ vs. initial shift $z_{0}$ for 8 RG iterations (symbols) using $4 \mathrm{SP}$. Dashed lines indicate linear fits

correctly. This fact underlines again the importance of the RG unit for a successful application of the RG approach.

\section{Conclusions}

The version of the network model [55] that has been most widely studied in the context of the $\mathrm{QH}$ effect, is the CC model [2], describing the electron motion in a disordered system in a strong magnetic field limit. The fact that the RG approach, within which the correlations between different scales are neglected, describes the results of the large-scale simulations of the CC model so accurately, indicates that only a few spatial correlations within each scale are responsible for the critical characteristics of the quantum Hall transition. More precisely, the structure of the eigenstates of a macroscopic sample at the transition can be predicted from the analysis of a single RG unit consisting of only five nodes. Further applications of this approach to the computation of the Hall resistance and the plateau-to-insulator transition shall be published elsewhere.

We thank B. Huckestein, M.E. Raikh, M. Schreiber, and U. Zülicke for stimulating discussions. This work was supported by the DFG within SFB393 and the priority research program on quantum Hall systems. Further support was provided by a DAAD-NSF collaborative research grant INT-0003710. 


\section{References}

1. B. Huckestein, Rev. Mod. Phys. 67, 357 (1995).

2. J. T. Chalker and P. D. Coddington, J. Phys.: Condens. Matter 21, 2665 (1988).

3. D.-H. Lee, Z. Wang, and S. Kivelson, Phys. Rev. Lett. 70, 4130 (1993).

4. B. Huckestein, Europhys. Lett. 20, 451 (1992).

5. P. Cain, R. A. Römer, M. Schreiber, and M. E. Raikh, Phys. Rev. B 64, 235326 (2001), ArXiv: cond-mat/0104045.

6. F. Hohls, U. Zeitler, and R. J. Haug, Phys. Rev. Lett. 86, 5124 (2001), ArXiv: cond-mat/0011009.

7. S. Koch, R. J. Haug, K. v. Klitzing, and K. Ploog, Phys. Rev. Lett. 67, 883 (1991).

8. D. Stauffer and A. Aharony, Introduction to Percolation Theory (Taylor and Francis, London, 1992).

9. A. G. Galstyan and M. E. Raikh, Phys. Rev. B 56, 1422 (1997).

10. D. P. Arovas, M. Janssen, and B. Shapiro, Phys. Rev. B 56, 4751 (1997), ArXiv: cond-mat/9702146.

11. A. Weymer and M. Janssen, Ann. Phys. (Leipzig) 7, 159 (1998), ArXiv: condmat/9805063.

12. M. Janssen, R. Merkt, J. Meyer, and A. Weymer, Physica 256-258, 65 (1998).

13. Z. Wang, B. Jovanovic, and D.-H. Lee, Phys. Rev. Lett. 77, 4426 (1996).

14. X. Wang, Q. Li, and C. M. Soukoulis, Phys. Rev. B 58, 3576 (1998).

15. Y. Avishai, Y. Band, and D. Brown, Phys. Rev. B 60, 8992 (1999).

16. B. I. Shklovskii, B. Shapiro, B. R. Sears, P. Lambrianides, and H. B. Shore, Phys. Rev. B 47, 11487 (1993).

17. J.-L. Pichard and G. Sarma, J. Phys. C 14, L127 (1981).

18. J.-L. Pichard and G. Sarma, J. Phys. C 14, L617 (1981).

19. A. MacKinnon and B. Kramer, Phys. Rev. Lett. 47, 1546 (1981).

20. A. MacKinnon and B. Kramer, Z. Phys. B 53, 1 (1983).

21. R. Landauer, Phil. Mag. 21, 863 (1970).

22. D. S. Fisher and P. A. Lee, Phys. Rev. B 23, 6851 (1981).

23. H. A. Fertig, Phys. Rev. B 38, 996 (1988).

24. R. Klesse and M. Metzler, Phys. Rev. Lett. 79, 721 (1997).

25. E. P. Wigner, Proc. Camb. Phil. Soc. 47, 790 (1951).

26. M. L. Mehta, Random Matrices and the Statistical Theory of Energy levels (Academic Press, New York, 1991).

27. M. Metzler, J. Phys. Soc. Japan 67, 4006 (1998).

28. M. Batsch and L. Schweitzer, in High Magnetic Fields in Physics of Semiconductors II: Proceedings of the International Conference, Würzburg 1996, edited by G. Landwehr and W. Ossau (World Scientific Publishers Co., Singapore, 1997), pp. 47-50, ArXiv: cond-mat/9608148.

29. W. H. Press, B. P. Flannery, S. A. Teukolsky, and W. T. Vetterling, Numerical Recipes in FORTRAN, 2nd ed. (Cambridge University Press, Cambridge, 1992).

30. F. Haake, Quantum Signatures of Chaos, 2nd ed. (Springer, Berlin, 1992).

31. H. Potempa and L. Schweitzer, J. Phys.: Condens. Matter 10, L431 (1998), ArXiv: cond-mat/9804312.

32. D. Braun, G. Montambaux, and M. Pascaud, Phys. Rev. Lett. 81, 1062 (1998), ArXiv: cond-mat/9712256. 
33. L. Schweitzer and H. Potempa, Physica A 266, 486 (1998), ArXiv: condmat/9809248.

34. Y. V. Fyodorov and A. D. Mirlin, Phys. Rev. B 55, 16001 (1997).

35. T. Kawarabayashi, T. Ohtsuki, K. Slevin, and Y. Ono, Phys. Rev. Lett. 77, 3593 (1996), ArXiv: cond-mat/9609226.

36. M. Batsch, L. Schweitzer, I. K. Zharekeshev, and B. Kramer, Phys. Rev. Lett. 77, 1552 (1996), ArXiv: cond-mat/9607070.

37. M. Feingold, Y. Avishai, and R. Berkovits, Phys. Rev. B 52, 8400 (1995), ArXiv: cond-mat/9503058.

38. T. Ohtsuki and Y. Ono, J. Phys. Soc. Japan 64, 4088 (1995), ArXiv: condmat/9509146.

39. M. Metzler and I. Varga, J. Phys. Soc. Japan 67, 1856 (1998).

40. M. Batsch, L. Schweitzer, and B. Kramer, Physica B 249, 792 (1998), ArXiv: cond-mat/9710011.

41. M. Metzler, J. Phys. Soc. Japan 68, 144 (1999).

42. Y. Ono, T. Ohtsuki, and B. Kramer, J. Phys. Soc. Japan 65, 1734 (1996), ArXiv: cond-mat/9603099.

43. S. Koch, R. J. Haug, K. v. Klitzing, and K. Ploog, Phys. Rev. B 43, 6828 (1991).

44. F. Hohls, U. Zeitler, and R. J. Haug, Phys. Rev. Lett. 88, 036802 (2002), ArXiv: cond-mat/0107412.

45. E. Abrahams, P. W. Anderson, D. C. Licciardello, and T. V. Ramakrishnan, Phys. Rev. Lett. 42, 673 (1979).

46. E. Hofstetter and M. Schreiber, Phys. Rev. B 49, 14726 (1994), ArXiv: condmat/9402093.

47. I. K. Zharekeshev and B. Kramer, Jpn. J. Appl. Phys. 34, 4361 (1995), ArXiv: cond-mat/9506114.

48. I. K. Zharekeshev and B. Kramer, Phys. Rev. B 51, 17239 (1995).

49. I. K. Zharekeshev and B. Kramer, Phys. Rev. Lett. 79, 717 (1997), ArXiv: cond-mat/9706255.

50. E. Hofstetter and M. Schreiber, Phys. Rev. B 48, 16979 (1993).

51. K. Slevin and T. Ohtsuki, Phys. Rev. Lett. 82, 382 (1999), ArXiv: condmat/9812065.

52. P. Cain, R. A. Römer, and M. E. Raikh, Phys. Rev. B 67, 075307 (2003), ArXiv: cond-mat/0209356.

53. M. Janssen, R. Merkt, and A. Weymer, Ann. Phys. (Leipzig) 7, 353 (1998).

54. U. Zülicke and E. Shimshoni, Phys. Rev. B 63, 241301 (2001), ArXiv: condmat/0101443.

55. B. Shapiro, Phys. Rev. Lett. 48, 823 (1982). 